\title{
The predictive value of cancer symptoms in primary care
}

GPs are presented, several times a day, with patients with a mass of intertwined symptoms. Many of these symptoms, often matted together like overcooked pasta, could be due to cancer. In practice most of them are not. However, neither GPs nor specialists have the experience to weight these symptoms accurately: GPs because they do not see enough patients with individual cancers, and specialists because they do not see enough patients who are healthy (leading to 'spectrum bias'1). For this reason 'gut feeling', instinct, or previous experience are all relatively blunt discriminators. Secondary care studies are also of limited use. What we really need are numbers from good primary care studies. These are surprisingly few and far between. ${ }^{2,3}$

Shapley et $a l^{3}$ analysed all high-quality primary care studies that identify clinical features that have, in at least one age/sex range, a positive predictive value (PPV) of $5 \%$ or more. In justifying their $5 \%$ figure they discuss what level of cancer risk should routinely be investigated.

The authors identify eight clinical features that predict cancer at the 5\% probability level: rectal bleeding, change in bowel habit, iron deficiency anaemia, macroscopic haematuria, a malignantfeeling prostate on rectal examination, breast lumps, and post menopausal bleeding. Unsurprisingly, age ranges are critical to PPV: iron deficiency anaemia in a 21-year-old female is extremely unlikely to be due to colorectal cancer whereas in a 60-year-old male cancer is likely. 'Change in bowel habit' is the only feature that obviously involves a subjective judgment by the doctor. ${ }^{2}$

The authors argue that, with a 5\% absolute risk of cancer, 'exclusion of cancer is obligatory unless exceptional circumstances exist'. They compare this level of cancer risk with the $5 \%$ reduction in 10 year cardiovascular risk expected for primary prevention with statins in individuals with a Framingham risk score of $20 \%$.
This is a useful 'back of the envelope' calculation. Quantifying absolute benefit is essential in assessing treatments. Quantifying absolute risk is also well developed in cardiovascular medicine. Yet quantifying absolute risk is a relative latecomer to the debate about cancer diagnosis. We often give patients numbers to enable them to make informed decisions about cardiovascular risk, but we rarely do so with cancer. How many women would have lifetime cervical smears if they knew that their chance of avoiding death is about 1 in 1000, while the chance of them having a cone biopsy is about 1 in $12 ?^{4}$

However, the authors' analysis clearly raises many questions that need more detailed analysis and debate: surely avoiding a $1 \%$ absolute risk in a 35 year old is at least as beneficial as a $10 \%$ risk avoided in a 65 year old. Is it as beneficial detecting a lung cancer (often incurable) as detecting a colorectal cancer (often curable); is there a net benefit with early detection of prostate cancer; and where do the patients' own views come into all of this?

What we actually want (as a minimum) are three bits of information: the PPV, the number needed to treat to save a life with early cancer treatment, and some assessment similar to a 'quality-adjusted life year' (QALY) for the whole process.

There are various reasons why these data are required fairly urgently. The obvious reason is that patients may be dying unnecessarily if we, as GPs, are initiating referral or investigation at too high a level of risk. The second reason is that inappropriate intervention at too low a level of risk is also harmful. As with randomised controlled trials, there is an asymmetry between the ease of detecting harm and detecting benefit. $^{5}$ Trials potentially measure the large benefit to the few patients who are diagnosed early and treated. They rarely measure the significant low level of harm to the large majority who are fruitlessly investigated. As GPs we are very aware of the risk of 'medicalising' people's lives and the potential cascade effect of over investigation. ${ }^{6}$

The last imperative is, I think, less well recognised. Referral guidance potentially has significant litigation costs associated with it if it is over inclusive. Much of the current National Institute for Health and Clinical Excellence referral guidance has been shown to have a sound evidence base. $^{7}$ However, some has little supporting data and is potentially problematic.

A GP may currently have difficulty justifying a failure to refer urgently a 60year-old male with a single prostatespecific antigen (PSA) of 6 (yet 10\% of males aged $50-70$ years have a raised PSA), or microscopic haematuria in a 60year-old female (yet reported prevalence rates are up to $16.1 \%^{8}$ ). The litigation bill for the NHS for delayed diagnosis of cancer is significant, and better data should help to make guidelines more robust and justifiable.

As virtually all cancer diagnoses start in primary care, it remains surprising that so little research is directed at this area. It would be very useful to repeat the methods of Shapley et al and identify current research that identifies clinical features with PPVs in the range of $1 \%$ to $5 \%$ and $<1 \%$.

The two most common methodologies for symptom-based research reviewed in this paper are retrospective cohort studies using clinical databases, such as the General Practice Research Database ${ }^{7}$ and primary care case control studies. ${ }^{9}$ Both methods are efficient and fruitful (although the retrospective cohort study may suffer from selective recording of symptoms in those referred with suspected cancer which erroneously elevates the measured PPV). It would also be useful to investigate whether there is a difference in PPV between volunteered 
symptoms (for example, volunteered dysphagia symptoms: 'My food sticks') and elicited symptoms ('Does your food stick?', 'Yes, a bit.').

Shapley et al's paper is very useful but it does highlight the pressing need for an explicit agenda for symptom-based research in primary care to inform cancer referral guidelines.

\section{Kevin Barraclough}

GP, Stroud.

\section{Provenance}

Commissioned; not peer reviewed.

\section{REFERENCES}

1. Jelinek M. Spectrum bias: why generalists and specialists do not connect. Evid Based Med 2008; 13(5): 132-133.
2. Hamilton W. Cancer diagnosis in primary care. $\mathrm{Br} J \mathrm{Gen}$ Pract 2010; 60(571): 121-128.

3. Shapley M, Mansell G, Jordan JL, Jordan KP. Positive predictive values of $\geq 5 \%$ in primary care for cancer: systematic review. Br J Gen Pract 2010; 10.3399/bjgp10X515412.

4. Raffle AE, Alden B, Quinn M, et al. Outcomes of screening to prevent cancer: analysis of cumulative incidence of cervical abnormality and modelling of cases and deaths prevented. BMJ 2003; 326: 901.

5. Kent DM, Hayward RA. Limitations of applying summary results of clinical trials to individual patients: the need for risk stratification. JAMA 2007; 298(10): 1209-1212.

6. Deyo RA. Cascade effects of medical technology. Annu Rev Public Health 2002; 23: 23-44.

7. Jones R, Latinovic R, Charlton J, Gulliford MC. Alarm symptoms in early diagnosis of cancer in primary care: cohort study using General Practice Research Database. BMJ 2007; 334(7602): 1040.

8. Cohen RA, Brown RS. Microscopic hematuria. N Engl Med 2003; 348(23): 2330-2338.

9. Hamilton W, Round A, Sharp D, Peters TJ. Clinical features of colorectal cancer before diagnosis: a population-based case-control study. Br J Cancer 2005; 93(4): 399-405.

DOI: 10.3399/bjgp10X515304

ADDRESS FOR CORRESPONDENCE

\section{Kevin Barraclough}

Hoyland House, Painswick,

Stroud, GL6 6RD, UK

E-mail:k.barraclough@btinternet.com 